\title{
Wild chromatin: regulation of eukaryotic genes in their natural chromatin context
}

\author{
Valerio Orlando ${ }^{1}$ and Katherine A. Jones ${ }^{2,3}$ \\ ${ }^{1}$ Dulbecco Telethon Institute, Institute of Genetics and Biophysics National Research Council, 80131 Naples, Italy; ${ }^{2}$ Regulatory \\ Biology Laboratory, The Salk Institute for Biological Studies, La Jolla, California 92037, USA
}

Nearly 40 years ago, François Jacob and Jacques Monod provided the first genetic evidence that genes are controlled through the opposing actions of activators and repressors on specific DNA targets. Although minimalistic, the classic paradigm provided by the regulation of the Lac operon in Escherichia coli quite accurately reflects the rationale of eukaryotic gene regulation, even in the context of highly organized chromosome structure. Transcriptional activation of most eukaryotic genes is now known to require several 10s of factors, many of which are responsive to developmental or environmental signaling pathways. Some regulators are enzymes that modify or reconfigure chromatin, whereas others influence the subnuclear organization of transcription factors or, in some cases, change the relative chromosomal environments of specific gene targets. Posttranslational modifications of histones or enhancer factors define localized chromatin domains as well as the activity and turnover of enhancer complexes. A crucial problem in the field now is to decipher how proper regulation springs from combinations of positive and negative factors, generally organized in multiprotein complexes or coupled to molecular engines, which recognize and modify regulatory loci embedded in chromatin.

Remarkably, after an early period in which nucleosomal DNA was seen as a negative and somehow passive template for transcription, chromatin and chromosome organization in the nucleus have now emerged as central parameters with direct and essential effects on the control of gene expression. The workshop "Regulation of Eukaryotic Genes in Their Natural Chromatin Context," hosted by Fundaciòn Juan March in Madrid, Spain (April 22-24, 2002), was organized by Miguel Beato and Ken Zaret to address some of the most pressing issues concerning how complex regulatory networks work in their natural molecular environment: the chromosome.

Histone modifications in the control of gene expression and genome plasticity

Contrary to the view that chromatin functions as a natural barrier to most DNA-dependent processes, new find-

${ }^{3}$ Corresponding author.

E-MAIL jones@salk.edu; FAX (858) 552-8285.

Article and publication are at http://www.genesdev.org/cgi/doi/10.1101/ gad.1017402. ings highlight a key role for modification of individual nucleosomes in gene regulation. Ultimately, the stability of open or closed chromatin is dictated by reversible histone tail modifications, which occur in patterns that correlate with gene activation or repression. A major task is to unravel how gene- and locus-specific patterns of modifications are initiated and maintained.

At the meeting, Dave Allis (University of Virginia, Charlottesville, VA, USA) showed that the Saccharomyces cerevisiae Rad6 gene product regulates methylation at Lys 4 (K4) on histone H3, often considered a hallmark of gene activation. Remarkably, RAD6 is not a histone methyltransferase but, rather, functions as a ubiquitinconjugating enzyme specific for histone H2B-K120 (K123 in yeast). Thus, ubiquitination of histone $\mathrm{H} 2 \mathrm{~B}$ is epistatic to the histone $\mathrm{H} 3-\mathrm{K} 4$ methylation. The converse is not true, namely, methylation at histone $\mathrm{H} 3-\mathrm{K} 4$ is not required for ubiquitination of histone $\mathrm{H} 2 \mathrm{~B}$. This example illustrates the importance of nucleosomal modification cross-talk, in which a modification at one site can profoundly influence modification at nearby sites or, remarkably, even on distant histone tails. Recent studies have suggested sequence similarities between histone deacetylase and deubiquitinating enzymes, which raises the intriguing possibility that histone deacetylase inhibitors such as trichostain A (TSA) might impact ubiquitindependent histone methylation pathways, which, in turn, affect histone acetylation. The Allis group have also found that phosphorylation at Ser 14 on histone H2B occurs selectively on apoptotic chromatin in vertebrates and may be mediated by Mst1 (mammalian sterile-like kinase), an apoptotic-inducing kinase that acts immediately downstream of caspase-3s.

Louis Mahadevan (University of Oxford, UK) addressed whether phosphorylation of histone $\mathrm{H} 3$ at S10 and acetylation at residues $\mathrm{K} 4$ and $\mathrm{K} 9$, which are both early events in the induction of the c-fos and c-jun genes, occur as concerted or independent events. Disruption of the MSK1 and MSK2 kinases resulted in the loss of S10 phosphorylation without affecting the overall levels of histone H3 acetylation, indicating that histone phosphorylation and acetylation may be regulated independently. These findings contrast with previous reports that phosphorylation at S10 precedes acetylation and gene activation. This group also reported that whereas 
H3 phosphorylation is an inducible event, acetylation is continuous and dynamic, even in quiescent cells and in the absence of transcription. The latter finding may be particularly relevant for maintenance programs, in which a locus specifies an appropriate level of acetylation through the regulated actions of opposing enzymatic activities. To define the function of different chromatin modifications in the various stages of mammalian X-chromosome inactivation, Bryan Turner (University of Birmingham, UK) presented immunohistochemical evidence that global changes in the acetylation of all four core histones occur in parallel and follow down-regulation (silencing) of X-linked genes in female ES cells. Remarkably, analyses using novel antisera capable of distinguishing di- and trimethylated lysine residues (H3-K4, $\mathrm{H} 3-\mathrm{K} 9$ ) revealed that $\mathrm{X}$ inactivation is accompanied by changes in the relative abundance of specific methylation modifications. Thus, although the inactive X-chromosome (Xi) lacks H3 dimethyl K4, H3 trimethyl K4, and $\mathrm{H} 3$ trimethyl $\mathrm{K} 9$, it retains $\mathrm{H} 3$ dimethyl $\mathrm{K} 9$ in amounts equivalent to those on the active X-chromosome (Xa). Evidence was presented that the relatively enhanced immunostaining of $\mathrm{Xi}$ with certain antisera to H3 methyl K9 is fixation-dependent, and likely reflects differences in chromatin conformation rather than increased levels of $\mathrm{H} 3$ methylation.

The mammalian genome undergoes extensive reprogramming after fertilization. The paternal genome is widely demethylated in the zygote, whereas the maternal genome is demethylated during cleavage division in the preimplantation embryo. After these epigenetic marks are removed, a wave of de novo methylation occurs postimplantation. Wolf Reik (Babraham Institute, Cambridge, UK) examined the possible relationship between DNA and histone methylation in preimplantation embryos. K9-histone $\mathrm{H} 3$ methylation is asymmetric at fertilization, and is immediately followed by DNA demethylation of the paternal genome. Later, de novo DNA methylation occurs, accompanied by global histone $\mathrm{H} 3-\mathrm{K} 9$ remethylation, specifically in inner-cellmass cells of the blastocysts. Reik also addressed why nuclear transplantation is such an inefficient process in animal cloning experiments. One explanation was suggested by looking at fibroblast nuclei implanted into enucleated oocytes, where immunolocalization studies revealed that implanted nuclei do not recapitulate the DNA and histone methylation patterns that are normally observed during early mouse embryogenesis.

A key goal in the field is the identification of evolutionarily conserved histone-specific methyltransferases (HMTs) that give rise to the epigenetic marks that impact genome stability and plasticity. Thomas Jenuwein (IMP, Vienna, Austria), examined knockout mice lacking both mammalian Su(var)3-9 HMTases (Suv39h1 and Suv39h2) and showed that these mice showed reduced viability and that the survivors acquire lymphomas at $\sim 30 \%$ penetrance. Remarkably, the constitutive heterochromatin in cells lacking both Suv39h genes was no longer reactive to antisera recognizing histone $\mathrm{H} 3$ Lys 9 (H3-K9) methylation, whereas the inactive X-chromo- some maintained H3-K9 methylation. This implies the existence in heterochromatin of more than one type of histone $\mathrm{H} 3$ methylation, and also indicates that more than one HMT controls the methylation state of heterochromatin. Whether histone lysine methyl groups are removed enzymatically is an open question at the present.

Much discussion focused on the fact that not all histone modifications are created equal. In particular, K4 and $\mathrm{K} 9$ of histone $\mathrm{H} 3$ can be mono-, di-, or trimethylated, and existing antisera do not appropriately distinguish these modifications. Should these distinct methylation states differentially impact gene regulation or chromatin compaction, the combinatorial power of signaling through histone modification would be enhanced significantly. Moreover, because most in vivo studies of histone modification rely on chromatin immunoprecipitation or immunolocalization, the quality and specificity of existing antibodies restricts our understanding of the state of native chromatin. Further, Louis Mahadevan (University of Oxford, UK) reported that second-site modifications in histone tails can strongly interfere with epitope recognition. In particular, he reported the case of a commercial K9/K18 acetyl H3 antiserum that could not detect the epitope if Ser10 is phosphorylated. In this case, therefore, phosphorylation at S10 would mask detection of histone $\mathrm{H} 3$ acetylation. Bryan Turner (University of Birmingham Medical School, Birmingham, UK) has shown that antibodies raised against dimethylated H3 tail peptides share little or no cross-reactivity with antibodies raised against the equivalent trimethylated peptides, and vice versa. This issue will deserve particular attention in the future, and as knowledge about complex histone modification profiles emerges, it will become crucial to develop reagents that more precisely define the state of regulated chromatin in vivo.

\section{Chromatin remodeling at activated genes in vivo}

An early step in gene activation involves activator-specific recruitment of energy-dependent nucleosome-remodeling and histone-modification complexes. Several distinct complexes, defined by the identity of the ATPase subunit, have been shown to disrupt nucleosomal arrays and open chromatin structure. Thus, SWI2/ SNF2 or ISWI-containing complexes, as well as histone acetyltransferases and methyltransferases are differentially recruited to specific subsets of genes through DNA-bound transcription factors. In this way, remodeling complexes recruited by one activator can facilitate binding and activation of other transcription factors that may be unable to recruit these enzymes. Similarly, enhancer factors may directly recruit specific histone acetyltransferases, methyltransferases, and ubiquitin ligases to modify local nucleosomes as well as the assembled enhancer complex. Protein modifications of enhancer complexes may control other steps in transcription, including elongation, reinitiation, and exchange of coactivators and corepressors.

Several reports presented at the workshop examined 
changes in nucleosome positioning and remodeling at highly induced genes in vivo, or effects of chromatin on the regulation of specific genes during development.

A fundamental question concerns how different chromatin remodelers participate in different biological processes. Hua-Ying Fan from Bob Kingston's lab (Harvard Medical School, Boston, MA, USA), presented evidence that two different ATP-dependent remodeling subunits, BRG1 and SNF2h (members of the SWI/SNF and ISWI families, respectively) produce distinctly remodeled intermediates at different rates. The continuous interconversion of remodeled states indicates that a regulated equilibrium between change of nucleosome position and rate of remodelling may contribute to the different roles of chromatin remodeling enzymes in vivo. Marco Bianchi (Department of Biological and Technological Research, Milan, Italy) demonstrated that the chromatinassociated HMGB1 protein facilitates nucleosome sliding and remodeling by ISWI. The HMGB1-induced loosening, or bulging, of DNA around the nucleosome could provide a linchpin between transcription factors and DNA.

Wolfram Horz (University of Munich, Germany) reported that acetylation of histone $\mathrm{H} 3$ by the yeast SAGA complex accelerates the rate of activation of the PHO5 promoter, but Gen 5 activity is not essential for its final activation. The Pho4 activator accumulated normally in nuclei in the absence of Gcn5 acetyltransferase activity. A similar delay in PHO5 activation and chromatin remodeling at the promoter is found in the absence of Snf2. Pho4 recruitment to the promoter is delayed under these conditions as shown by ChIP analysis. Thus, nucleosome acetylation and remodeling function to reinforce a rapid response to activation, but are not essential to reach normal PHO5 transcription levels at much later stages in induction. Ernesto Di Mauro (University La Sapienza, Rome, Italy) examined nucleosome destabilization upon activation of the $A D H 2$ gene in $S$. cerevisiae. High-resolution analysis of nucleosome positioning revealed that nucleosomes -1 and +1 surrounding the site of initiation change positioning by a few nucleotides in the direction of transcription upon induction. These changes require the ADR1 activator and are also observed with mutants with impaired RNAP II catalytic activity, indicating that nucleosome repositioning is not a result of ongoing transcription.

Dramatic effects of nucleosome positioning are also observed upon induction of the MMTV promoter by steroid hormones. Miguel Beato (CRG/UPF, Barcelona, Spain) showed that the progesterone receptor recruits ATP-dependent remodeling activities, which enable binding of the enhancer factor NF1. NF1 binding stabilizes an open chromatin conformation that facilitates binding of additional receptors. In vitro minichromosome assembly experiments suggest that linker histone $\mathrm{H} 1$ improves nucleosome positioning and enhances transcriptional synergism between hormone receptors and NF1, which is preceded by phosphorylation of histone $\mathrm{H} 1$ and its depletion from the MMTV promoter. Trevor Archer (NIEHS, Research Triangle Park, NC, USA) ex- amined how steroid hormone-inducible genes become refractory to further stimulation following induction. He showed that the SWI/SNF chromatin-remodeling complex is recruited by the glucocorticoid receptor (GR) in a hormone-dependent manner via interactions with a SWI/SNF subunit, termed Brahma-associated factor 60a (BAF60a). Further, expression of a mutant form of BAF60a binds the glucocorticoid receptor (GR) in a hormone-dependent manner and inhibits the activity of BRG1-containing remodeling complexes. Thus, the hormone-activated GR may engage SWI/SNF through BAF60a to promote transient remodeling. Archer's lab also investigated the role of histone $\mathrm{H} 1$ phosphorylation in the refractory state, and showed that inhibition of histone $\mathrm{H} 1$ kinase $\mathrm{CD}-\mathrm{K} 2$ reduces MMTV transcription in vivo. Using a mass spectrometric approach, they showed that prolonged treatment with dexamethasone led to dephosphorylation of a specific $\mathrm{H} 1$ isoform (H1.3) concomitant with repression. Through the use of photobleaching experiments, Gordon Hager (National Institutes of Health, Bethesda, MD, USA) examined the behavior of tagged enhancer factors bound at the MMTV promoter, and discovered that most enhancer factors are rapidly displaced from MMTV DNA. Whereas DNAbound factors appear to dissociate within seconds, the stably recruited RNAPII remains associated far longer with the template. In vitro, Hager found that GR recruits a SWI/SNF complex, but is actively displaced from the MMTV chromatin template during remodeling, simultaneous with a transient burst of chromatin modifications, including $\mathrm{H} 3$ acetylation and methylation, during activation. The dynamics of DNA repair in chromatin was illustrated in a talk from Fritz Thoma (Swiss Federal Institute of Technology-Honggerberg, Zurich, Switzerland), who used photolyase as a molecular tool to compare the kinetics of repair (NER) of DNA lesions with the slower nucleotide excision repair mechanism in yeast euchromatin and heterochromatin. The Thoma group find that active genes are more rapidly repaired than silenced genes, which is consistent with a more compact chromatin structure of silenced genes. Interestingly both photolyase and NER appear to have open access to nucleolar chromatin.

The problem of how to establish and maintain promoter competence during development was addressed by Ken Zaret (Fox Chase Cancer Center, Philadelphia, PA, USA). Two enhancer factors, GATA-4 and HNF3 (FoxA), bind the albumin enhancer at 8.5-11.5 d of mouse development in the endoderm, prior to binding of other factors responsible for liver-specific albumin gene expression. These two factors are sufficient to trigger an opening of the chromatin structure that supports the binding of other regulatory factors, and are lost in cells that are not committed to differentiate to liver. HNF3 can interact directly with the $\mathrm{H} 3$ and $\mathrm{H} 4$ core histones, and binding is correlated with opening of chromatin structure, although not mediated directly through the histone tails. Taken together, these data suggest that specialized transcription factors can create preset conditions that allow a gene to undergo subsequent rapid activation. The roles 
of different coactivators in the developmentally regulated Wnt and Notch enhancer complexes was discussed by Kathy Jones (The Salk Institute, La Jolla, CA, USA). The Wnt-associated coactivator $\beta$-catenin strongly enhances binding of the LEF-1 HMG protein to chromatin and supplies an activation domain sufficient to support transcription initiation on chromatin in vitro. In contrast, the Notch intracellular domain (ICD) bound to the CSL enhancer-binding protein was unable to support transcription in vitro in the absence of a third coactivator, Mastermind. Mastermind was also shown to couple activation with disassembly of the Notch enhancer complex and to facilitate phosphorylation in vivo of two associated factors, the Notch ICD and CBP/p300, causing the latter to redistribute to large foci in cells expressing Mastermind. Emerging studies from other systems have suggested a key role for protein phosphorylation and ubiquitination in coupling of activation with disassembly of activator complexes, and can impact the timing and progression of transcription initiation and subsequent steps.

\section{The organization and function of nuclear domains}

Several talks highlighted important advances in defining the effects of long-range regulatory elements and subnuclear positioning on the control of gene expression. Gary Felsenfeld (National Institutes of Health, Bethesda, MD, USA) discussed the action of insulators, which can shield enhancers from the actions of external promoters and prevent condensed chromatin from spreading into active gene loci. Studies of the chick $\beta$-globin $5^{\prime}$ insulator showed that a regulatory DNA-binding protein, CTCF, mediates enhancer blocking activity but does not convey protection against position effects resulting from different sites of integration. Remarkably, CTCF also functions at the Igf2 boundary element, and sites for this factor lie near Xist and may contribute to X-chromosome inactivation. Analysis of the modification state of nucleosomes within the insulated transgene suggested a model in which insulators directly facilitate nucleosome acetylation, and that the resulting open chromatin structure supports the binding of factors that protect the gene against DNA methylation. A new zinc finger protein, termed BGP1, was shown to bind to G-string elements in the insulator and may be involved in protecting against position effects. Frank Grosveld (Erasmus University, The Netherlands) discussed models for stochastic gene activation, which have been invoked to explain monoallelic gene expression patterns as well as other aspects of gene activation. Analysis of allelic transcription patterns and mRNA levels in single cells showed an imbalance of $\alpha$-globin versus $\beta$-globin gene expression, indicating that once a cell chooses a particular pattern of expression, the pattern becomes fixed in that cell. The findings emphasize the importance of stochastic events in the phenotypic differences that are observed in otherwise genetically identical cells or organisms.

Susan Gasser (University of Geneva, Switzerland) described a series of elegant studies showing that the po- sitioning of chromosomal domains within the interphase nucleus facilitates transcriptional repression in yeast. Analysis of GFP-tagged chromosomes in interphase yeast cells by time-lapse microscopy revealed that internal tagged sites along yeast chromosomes are highly mobile in $G_{1}$ phase. This rapid chromosome movement is ATP-dependent and linked to the metabolic state of the cell, and becomes constrained in S phase. In contrast, chromatin movement is constrained at telomeres and centromeres in both $G_{1}$ and $S$ phases, when these elements are anchored near the nuclear envelope. The perinuclear attachment of telomeres requires the heterodimeric Ku complex and an unidentified nuclear envelope component. Importantly, a second, Sir-dependent, pathway seems to tether natural telomeres at the nuclear periphery in S phase. Because previous work has shown that yeast cells must pass through $S$ phase to establish heritable silent chromatin, this S-phase-dependent tethering correlates with subtelomeric repression and may provide a mechanistic link between repression and anchorage. Another interesting possibility suggested by these studies is that silent chromatin may play a direct role in the anchoring of telomeres.

Peter Becker (Ludwig-Maximilians University, Munich, Germany) discussed the targeting of the histone acetyltransferase MOF to the male X-chromosome for dosage compensation in flies. Dosage compensation in flies involves the increase in expression of X-chromosome genes in the male, as both sexes must express equivalent levels of X-chromosome genes. Proper dosage compensation requires the epigenetic formation of stable chromatin structure and the coating of the X-chromosome by an X-encoded, noncoding regulatory RNA. The hyperactive male $\mathrm{X}$-chromosome was shown to be acetylated at histone H4-K16 by the MOF histone acetyltransferase, a member of the MYST family that is targeted to the X-chromosome as part of the dosage-compensation complex. This complex also includes the noncoding roX RNA and several male-specific lethal proteins including the MLE helicase and a histone H3 kinase. The Becker lab is taking a biochemical approach to define how the dosage-compensation complex is assembled and targeted to the X-chromosome, and have further elaborated on the roles of the MLE helicase, MOF, and MSL-3 in binding to roX RNA. Although MOF contains a chromodomain related to those of other proteins that recognize methylated histones, this region of MOF has been implicated to play a role in binding to RNA. Becker reported on the role of a conserved $\mathrm{C} 2 \mathrm{HC}$ zinc finger module for interaction of MOF with the nucleosomal substrate. In collaboration with the Kuroda Laboratory (Houston, TX, USA), the Becker Laboratory has mapped an X-chromosomal dosage-compensation complex entry site to a short DNA fragment. Using chromatin immunoprecipitation (X-ChIP) they are presently searching for additional high-affinity sites for the complex. The goal is to define the principles that allow the targeting and coating of the X-chromosome by epigenetic regulators. Keiko Ozato (National Institutes of Health, Bethesda, MD, USA) discussed the regulation of the mammalian double 
bromodomain-containing BET gene family. The bromodomain is a chromatin-targeting module found in many nuclear factors that mediates binding to acetylated histone H4. Histone deacetylase inhibitors were found to stabilize the binding of BRD4 to acetylated chromatin, and fluorescence resonance energy transfer (FRET) experiments with coexpressed tagged histone $\mathrm{H} 4$ revealed colocalization to noncentromeric chromatin on mitotic chromosomes. Ectopic expression of BRD4 blocked the $\mathrm{G}_{1} / \mathrm{S}$ transition, and BRD4 was shown to interact directly with DNA replication factor C (RFC140) in a manner that correlates with inhibition of DNA replication and entry to S phase. These findings indicate that BRD4 may recruit the RFC to acetylated chromatin or affect the timing of RFC activation during $S$ phase.

\section{Heterochromatin: open or closed?}

Cell identity is largely determined by cell-specific transcription profiles. After determination, gene-specific silencing is maintained and transmitted to daughter cells by epigenetic mechanisms involving chromatin modification and subnuclear compartmentalization. In Drosophila, the Polycomb group proteins (PcG) control cell identity by maintaining homeotic and other selector genes in a repressed state. PcG proteins act as multiprotein complexes that act via specialized DNA elements called Polycomb response elements (PREs). PREs are thought to maintain silencing by driving the building-up of heterochromatin-like structure. Details of the mechanism by which PcG maintains silencing are largely unknown.

Valerio Orlando (Telethon and Institute of Genetics and Biophysics, National Research Council, Naples, Italy) examined in vivo the role of PcG at promoters and reported that PcG-repressed chromatin does not exclude general transcription factors, but, rather, contains a reactivatable RNAPII complex. The bithorax complex repressed promoters look similar to active promoters in that they contain K4 (not K9) methylated H3, hyperphosphorylated RNAPII, and elongation factors. Furthermore, the PcG proteins were shown to coimmunoprecipitate with elongation factors and cyclin $\mathrm{T}$, suggesting that the block to transcription lies downstream of phosphorylation of the RNAPII CTD. In Drosophila, at least two major PcG protein complexes have been characterized; an early complex (ESC-EZ complex), which is part of a determination cascade that establishes the homeotic gene transcription state, and a later (PRC1) complex, which acts as the memory system. An unsolved key issue is how the memory system reads the promoter state in early embryogenesis, and then sets up the memory lock. Vincenzo Pirrotta (University of Geneva, Switzerland) showed that the ESC-EZ complex contains histone methyltransferase activity and that a burst of histone acetylation (provided by transient GCN5 expression) in early development impairs methylation and maintenance in a PRE-reporter construct. Also in this case, a stalled RNAPII was reported to sit at repressed promoters. In the context of gene silencing and heterochroma- tin, David Gross (Louisiana State University Health Sciences Center, New Orleans, LA, USA) showed that in the yeast $S$. cerevisiae silent, SIR-generated heterochromatin is permissive to constitutive-binding transcription factors, TBP, and both hypo- and hyperphosphorylated RNAPII. Moreover, the Sir2 and Sir3 proteins remain bound to the promoter even under conditions of robust transcription. The heat-shock transcription factor HSF can override silencing in the absence of either the Sas3 or Gcn5 acetyltransferases, suggesting that histone acetylation is not a prerequisite step in release of heterochromatic gene silencing. On the other hand, SWI/SNF is required, indicating a role for ATP-dependent chromatin remodeling.

\section{Perspectives}

This conference focused on understanding how DNAdependent processing events function in the context of native chromatin structure and nuclear organization, particularly as defined by physiological regulatory pathways. Collectively, the talks highlighted chromatin as a programmable substrate that regulates the assembly of molecular engines, depending on its local modification profile. A continued theme is the importance of individual histones and specific covalent modifications in the formation and stability of these protein-protein interactions. Because nucleosomes are major targets of cell signaling, gene-specific histone modification pathways will have to be precisely identified and deciphered. Recent technical developments, such as chromatin immunoprecipitation and high resolution in vivo genomic analysis, have opened new possibilities for addressing the role of chromatin structure and its modifications. Other key parameters are the dynamics of nucleosome positioning and the importance of higher-order chromatin structure in regulating gene loci. In some systems, the packaging of DNA into chromatin determines which regulatory proteins interact with their cognate elements and, thus, the outcome of the transcriptional response. It now appears that many genes are preset for activation, and that transcriptional activators as well as general transcription factors may reside on the DNA and become activated only later, for example, in response to specific signaling pathways. Tissue-specific promoters appear to be preset by transcription factors, giving genes the chance to be switched according to developmental programs. As many of these factors regulate more than one gene, one can envisage that pathway-specific transcription complexes sit on the chromosome and can be coordinately switched. Genome-wide identification of target genes by ChIP will provide global views of these dynamics. This is true also for putatively heterochromatinized promoters regulated by the SIR and Polycomb proteins, and indicate that silenced, developmentally regulated genes may retain the ability to be reactivated at all times, which has intriguing implications for cell reprogramming. Beyond the widespread role of histone modification, posttranslational modifications of enhancer fac- 
tor complexes can impact the activity, localization, and timing of transcription for tightly regulated genes.

As always, continued progress relies on novel specific technical advances and overcoming existing technical limitations. For example, it is becoming clear that opposing forces trafficking on chromatin represent the sum of events and interactions that may take only a few seconds, whereas conventional ChIP protocols fix cells for minutes. Thus, time is clearly a fundamental parameter in gene regulation, especially in light of stochastic events. Similarly, it is necessary to define where determined events take place within the nucleus. An accurate understanding of all dimensions of native chromatin also directly impacts on efforts to model regulation through biochemical reconstitution studies. The continued application of novel approaches and concepts such as those described at the meeting, and the further evolution of new techniques such as laser cross-linking and live cell imaging, will, therefore, make chromatin biologists happier in their natural context.

\section{Acknowledgments}

The Fundación Juan March provided an interactive setting conducive for thoughtful talks and discussions. We are grateful to Ken Zaret and Miguel Beato for conceiving and hosting this stimulating meeting, and to Andrés Gonzáles and Lucia Franco for their outstanding management of the workshop as well as to all of our colleagues who participated in Madrid. 


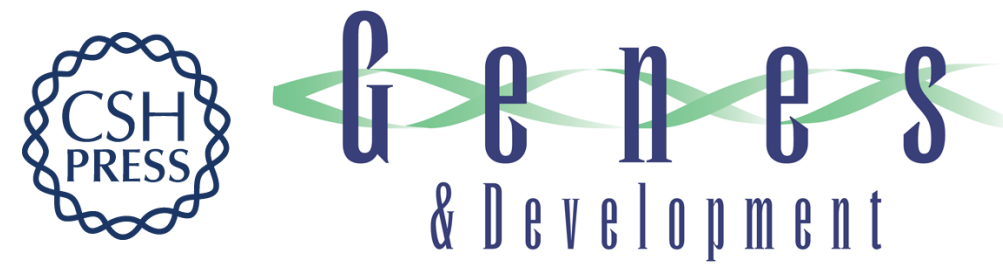

\section{Wild chromatin: regulation of eukaryotic genes in their natural chromatin context}

Valerio Orlando and Katherine A. Jones

Genes Dev. 2002, 16:

Access the most recent version at doi:10.1101/gad.1017402

\section{License}

Email Alerting

Service

Receive free email alerts when new articles cite this article - sign up in the box at the top right corner of the article or click here.

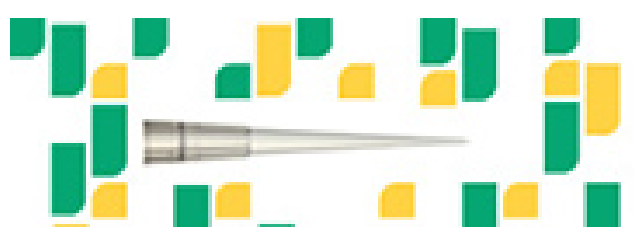

Focused on your science. 